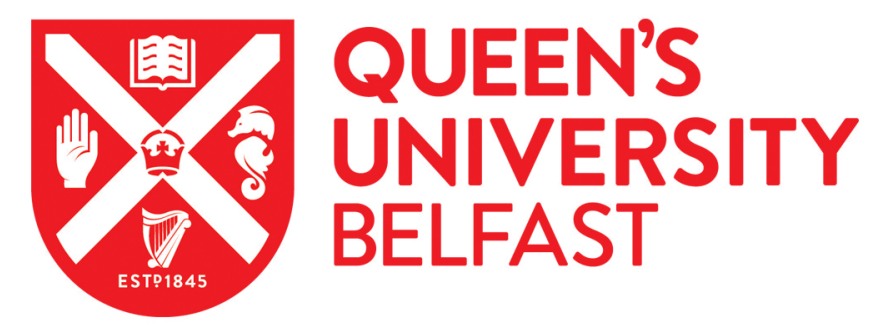

\title{
A Multifaceted Vigilare System for Intelligent Transportation Services in Smart Cities
}

Kumar, R., Goel, S., Sharma, V., Garg, L., Srinivasan, K., \& Julka, N. (2020). A Multifaceted Vigilare System for Intelligent Transportation Services in Smart Cities. IEEE Internet of Things Magazine.

https://doi.org/10.1109/IOTM.0001.2000041

Published in:

IEEE Internet of Things Magazine

Document Version:

Peer reviewed version

Queen's University Belfast - Research Portal:

Link to publication record in Queen's University Belfast Research Portal

Publisher rights

Copyright 2020 IEEE. This work is made available online in accordance with the publisher's policies. Please refer to any applicable terms of use of the publisher.

\section{General rights}

Copyright for the publications made accessible via the Queen's University Belfast Research Portal is retained by the author(s) and / or other copyright owners and it is a condition of accessing these publications that users recognise and abide by the legal requirements associated with these rights.

Take down policy

The Research Portal is Queen's institutional repository that provides access to Queen's research output. Every effort has been made to ensure that content in the Research Portal does not infringe any person's rights, or applicable UK laws. If you discover content in the Research Portal that you believe breaches copyright or violates any law, please contact openaccess@qub.ac.uk. 


\title{
A Multifaceted Vigilare System for Intelligent Transportation Services in Smart Cities
}

\author{
Ravinder Kumar, Shubham Goel, Vishal Sharma, Lalit Garg, Kathiravan Srinivasan, \\ Neeraj Julka
}

\begin{abstract}
Internet of Things (IoT), Information and Communication Technology (ICT), and data mining technologies are three prime pillars of any smart city project. The intelligent urban transportation system is an indispensable part of any smart city and acts as a major contributor to a city's vitality. An extensive amount of vehicular information is being amassed by Wireless Sensor Networks (WSNs), Vehicular Ad Hoc Networks (VANETs), and Radio-Frequency Identification (RFID) based Intelligent Transportation System (ITS), which can provide an insightful image of various ITS issues concerning safety, security, and management. Merely depending on WSN and RFID predicated technologies for the construction of ITS is not adequate to realise smart city dream in developing countries due to technological and monetary barriers. Considering this, an Intelligent Vigilare System (IVS) for ITS is proposed, which sanctions network implementation without disrupting the current vehicular infrastructure through multifaceted modeling.
\end{abstract}

Index Terms-Smart City, Intelligent Transportation Systems, Urban Computing, Vehicular Networks.

\section{SMART Cities AND InTELligent TRANSPORTATION SYSTEM (ITS)}

A smart city provides a platform for global urbanization, where Information and Communication Technology (ICT) and the Internet of Things (IoT) are used to support the innovations. Smart solutions like green building, intelligent traffic management, security systems, modern industrial control system, vehicle tracking, and ambient assisted living, are some of the prime targets under smart city

R. Kumar and S. Goel are with the Thapar Institute of Engineering and Technology, Patiala-147004, India; ravinder@thapar.edu, shubham.12789@gmail.com. V. Sharma is with EEECS, Queen's University Belfast, United Kingdom; vishal_sharma2012@hotmail.com. L. Garg is with the Faculty of Information \& Communication Technology University of Malta, Malta; lalit.garg@um.edu.mt. K. Srinivasan is with the School of Information Technology and Engineering, Vellore Institute of Technology, Vellore; kathiravan.srinivasan@vit.ac.in. N. Julka is with ECE Department, SLIET, Longowal, Punjab, India; neerajjulkasliet@gmail.com. projects $[1,2]$. Smart cities make use of numerous sensors of different types for collecting data pertaining to different aspects of the city at a commonplace, for effective and smart governance. Therefore, a smart city is often called a digitally interconnected and interoperable city. Intelligent Transportation System (ITS) is among the key aspects of any smart city project $[3,4]$, without which every claim of constructing an international city, providing higher standards of livings to its citizens, becomes groundless. It is not a standalone system but a joint venture of IoT, ICT, and data mining, where IoT and ICT are used to obtain and collect real-time data by utilizing various communication standards and technology enablers [5-7]. It also involves forecasting the traffic-flow with on-road sensors [8].

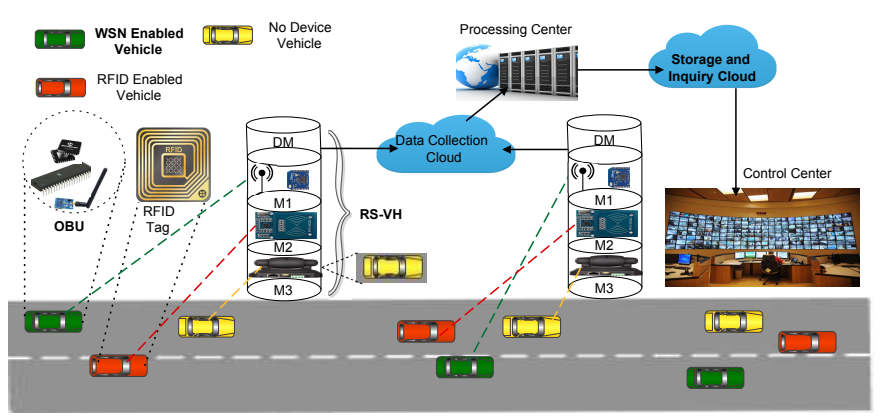

Fig. 1: An exemplary illustration of the proposed IVS Framework.

Over the past few years, Wireless Sensor Network (WSN) and Vehicular Ad Hoc Network (VANET) based approaches have been used for the construction of ITS with effective results in some developed countries, but constructing the ITS using only these technologies cannot guarantee a realtime solution [9]. Despite being advantageous, the conditions for adopting WSN and VANET based technologies are completely different in different scenarios. The biggest difference lies in terms of 
population size, the money required, and technology support. The need to embrace the benefits of ITS and a multi-modular solution to fulfill it without disrupting the current vehicular system for ITS has motivated the idea discussed in this article.

\section{Multifaceted Intelligent Vigilare SYSTEM}

The proposed approach depends on multiple technologies that are used to conceptualize an Intelligent Vigilare System (IVS) which can act as a multifaceted solution for the issues involved in the building of an efficient ITS. A simplified framework of the proposed IVS for implementing ITS in the smart cities is provided in Fig. 1. IVS is an infrastructure-based system of Road Side - Vigilare Hubs (RS-VH). Each RS-VH is equipped with a Radio-Frequency Identification (RFID), sensors, and Camera. These RS-VHs must be installed on a roadside of the entire city to collect real-time traffic data. RFID and WSN sensors are used to collect data from the vehicles which are equipped with an On-Board Unit (OBU). But for the vehicles which are incapable of supporting OBU technology, to capture their state information, the RS-VH camera facility is used.

The proposed system provides a common platform for WSN, RFID, and Image processing-based technologies to collectively contribute to the development of ITS. In WSN or VANETs -based ITS, each vehicle must be equipped with an OBU. Many vehicle manufacturers have started providing OBU, like facilities in their new models as per the demand of the world motor vehicle market. However, for providing a generic solution and supporting the existing vehicles, adequate solutions are necessary to avoid excessive overheads of old vehicles. This problem becomes severe for developing countries where the cost of installations and the number of old vehicles are too high. Therefore, it is better to adopt some other feasible and ubiquitous technologies for building an ITS without disrupting current vehicular operations, especially in developing countries. The details of each module used in the proposed model are presented in the following subsections.

\section{A. WSN modules}

WSN modules use a network of devices, equipped with a special type of sensors and microcontrollers

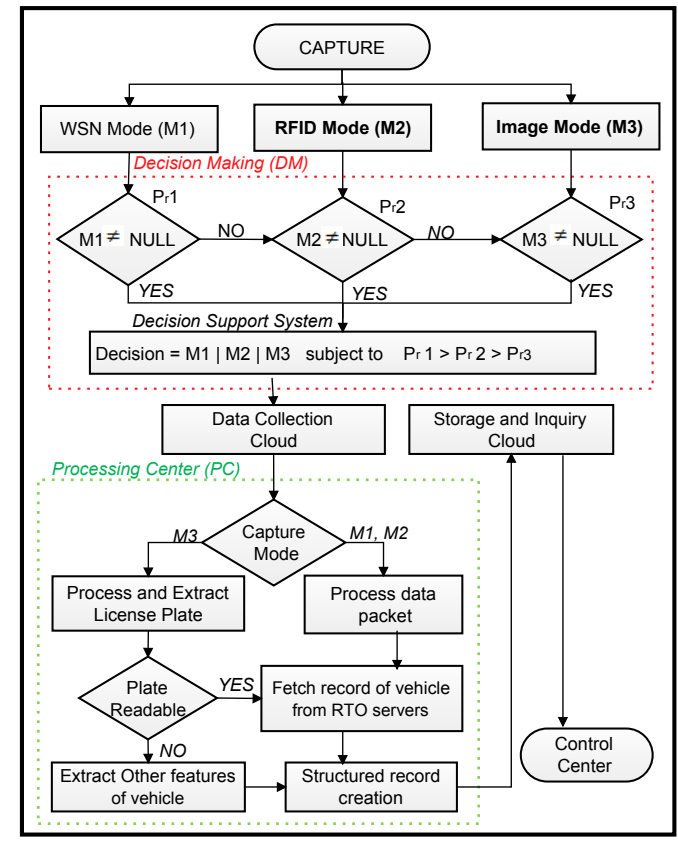

Fig. 2: A detailed workflow and Data-flow of IVS modules.

that can communicate the gathered information to each other over a wireless link. Different devices are available to provide wireless communication from Vehicle to Vehicle (V2V) and Vehicle to Infrastructure (V2I), some of which are successfully deployed in some developed countries for V2I communications. However, to the best of our knowledge, there is hardly any concrete deployment evidence available for the pure V2V system and the majority of them are in their initial phase of development.

Even in the proposed IVS, V2I based wireless communication is performed as some real-time examples are available for reference. WSN is configured by embedding a ZigBee transceiver, working on the principals of ZigBee protocol designed over the specifications of the IEEE 802.15.4 standard, into RS-VH and vehicles. For the sake of simplicity, it is assumed that the ZigBee transceiver is the standard transceiver to be provided in the OBU for a vehicle, by the manufacturer. A micro-controller together with a pre-programmable chip i.e. Erasable Programmable Read-Only Memory (EPROM) is also embedded into the OBU. An EPROM is used to store a special data packet i.e. "Who am $i$ " packet that is coded by a vehicle manufacturer. This packet will encapsulate each and every detail of the vehicle. The micro-controller is used for the management of the data packet as per the instructions of ZigBee 
transceiver.

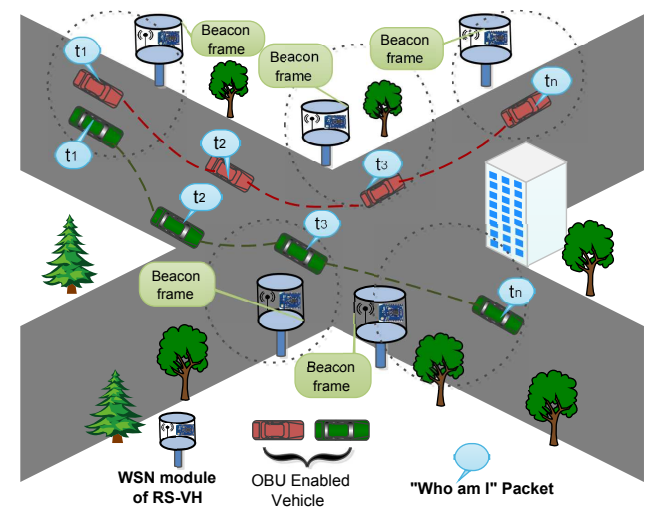

(a)

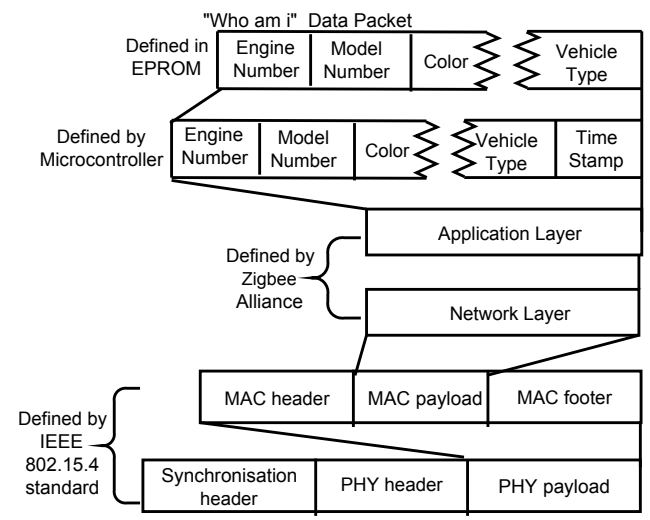

(b)

Fig. 3: (a)WSN-Based Communications in IVS, (b) Zigbee Protocol stack

The ZigBee transceiver present in RS-VH of IVS is responsible for establishing its WPAN where the network boundaries are up to the transmission range of the transceiver i.e. 10 meters usually. To collect the information of vehicles in IVS, a communication is initiated by the RS-VH's transceiver where a beacon frame is continuously broadcasted after a predefined interval of time. It contains the unique ID of RS-VH i.e. RS-VH_id and other information which is required by a vehicle's transceiver to become the part of WPAN.

Before handing over the packet to the vehicle's transceiver, a micro-controller will append the current timestamp of the system with the packet, as shown in Fig. 3(b). The timestamp will provide the time derivative proof of the vehicle's locations as illustrated in Fig. 3(a), where $t_{1}, t_{2}, \ldots, t_{n}$ timestamps encapsulated in respective "Who am $i$ " packets are providing complete trajectory for both red and green cars. After embedding the timestamp, a packet is passed through the different layers of the protocol stack, as shown in Fig. 3(b). In IVS, the beacon frame can only be sent by the RS-VH and the data frame can only be sent by a vehicle to the $\mathrm{RS}-\mathrm{VH}$ in a WPAN. There is no communication between peer vehicles in a WPAN. After receiving the packet at RS-VH, it is de-capsulated and handed over to the Decision Module (DM) in order to take a decision on data which is to be uploaded into the data collection cloud.

\section{B. RFID module}

RFID system consists of two main components, i.e. an RFID reader and the RFID tag. In the proposed IVS, the RFID reader module is installed into the RS-VH just like a ZigBee transceiver and a pre-programmable RFID tag i.e. $P_{p}^{t}$ is mounted on the windscreen of a vehicle. The engine number of a vehicle is encoded into $P_{p}^{t}$. Radio waves are continuously being emitted by an RFID reader and whenever a vehicle enters the transmission range of an RFID reader then $P_{p}^{t}$ will send the encoded information as a feedback signal. Once the signal is interpreted by the RFID reader module of the RS$\mathrm{VH}$, then it is handed over to DM.

\section{Image-based recognition module}

Merely depending on WSN and RFID based technologies for the construction of ITS in smart cities is not enough. Most of on-road vehicles are incompatible with these technologies and a lot of money is required to implement WSN and RFID based standards. Image and video processing are getting very much attention from researchers to build transport-related applications like front vehicle tracking [10], number plate recognition, collision avoidance, etc. For a real-time vision system, a computationally lightweight algorithm is designed [11], which can track tail lights of front vehicles and detect the motion parameters like brakes, turns, etc. In the image mode of IVS, two different approaches are followed to uniquely identify an on-road vehicle. The first one is the vehicle's license plate recognition and the second is to identify the vehicle based on its other broader features like color, brand logo, shape, etc. The results obtained by the second approach are less precise in comparison to the first one but the precision can be increased by considering more features for vehicle identification. The second approach is only used when it is not possible 
to recognize the license plate of a vehicle due to various reasons like broken, dirty, or unavailability of the plate and poor image quality.

In both the approaches, the image passes through a hierarchy of image manipulation phases to finally identify the vehicle. The foremost phase for both approaches is to capture the images of a vehicle using a bidirectional colored camera having a shutter speed of at least $1 / 1000$. To ensure that the images taken by the camera are free from any distortion or blurring effect, a global shutter is used in place of a rolling shutter. As the object whose image has to be taken is not still, a slow and rolling shutter can result in a blurred image that is of no use for an efficient license plate recognition system. A distance measuring sensor is embedded into $\mathrm{RS}-\mathrm{VH}$ to activate the camera whenever a vehicle is at the predefined distance $d$ from the RS-VH. The camera remains active to capture the images until a deactivate signal is received from the second sensor, which measures the distance of a vehicle that had already crossed the RS-VH node. Both the front-back images of the vehicles are captured by a bidirectional camera. The images, current timestamp, and the RS-VH_id are then encapsulated into a packet for the DM.

\section{Decision Module (DM)}

The decision about the vehicle information to be uploaded into the data collection cloud is taken by the DM of the RS-VH. The Decision Support System present inside the RS-VH will analyze the vehicle information packets corresponding to WSN, RFID, and Image modes. The sequence in which the packets are analyzed, to inspect that the packet is valid or invalid, is in the order of their assigned priorities i.e. $P_{r}^{1}, P_{r}^{2}$, and $P_{r}^{3}$ for WSN, RFID, and Image mode, respectively. The valid packet refers to not NULL packet i.e. information other than RSVH_id and the timestamp is also present inside the packet. The moment at which a valid packet is identified by the DM, it stops further analysis and uploads it into the temporary storage called a Data Collection Cloud. The detailed logical operations in the DM can be observed from Fig. 2.

\section{E. Processing Center (PC)}

The Processing Center (PC) involves a background center where the vehicle information packets are continuously processed to generate a structured record. The decision about the type of processing sequence required to obtain the knowledge from the packet depends on the capture mode, as shown in Fig. 2. For both M1 and M2 modes, a similar type of processing is required to extract the vehicle's identity along with the RS-VH_id, and timestamp. After getting the vehicle identity, other details are then obtained from the Regional Transport Office (RTO) servers. A structured record for the vehicle is then created using vehicle information, the RSVH_id, and timestamp. In the case of mode M3, firstly, the techniques of image processing are applied to recognize the license plate of a vehicle, which is passed through a series of image manipulation phases to uniquely identify the vehicle in the last phase. The foremost phase i.e. obtaining a high-quality image of the on-road vehicle is already performed by $\mathrm{RS}-\mathrm{VH}$. The remaining set of manipulation phases can be broadly divided into two categories i.e. detection and recognition. License plate detection refers to the extraction of the image region which consists of the plate, while plate segmentation and character identification fall under-recognition category. The performance of the entire system is greatly influenced by the accuracy of techniques chosen for license plate detection. Out of the well-known techniques, bounding box or edge detection technique is found to be more productive in terms of computations required and ubiquity. In the futuristic IVS, the advantage of the high dimensionality handling and discriminating ability of Convolution Neural Networks (CNN) can be taken to perform character and edge-based plate detection[12]. The concept of projections can also be used for horizontal and vertical edge detection.

\section{F. Caching and Secure Seeding for IVS}

Caching and security of operations help to facilitate the performance of any system. In the proposed IVS, both of these are provided depending on the module under which the data is collected from the vehicles. The caching is performed globally as well as locally depending on the classification of DM into M1, M2, or M3. The device-wise caching helps to fasten the exchange of information between the intra-DM facilities. However, the overheads of parsing the incoming traffic are one of the issues to be resolved, which can be handled through priority packets as expressed in the previous section. A 


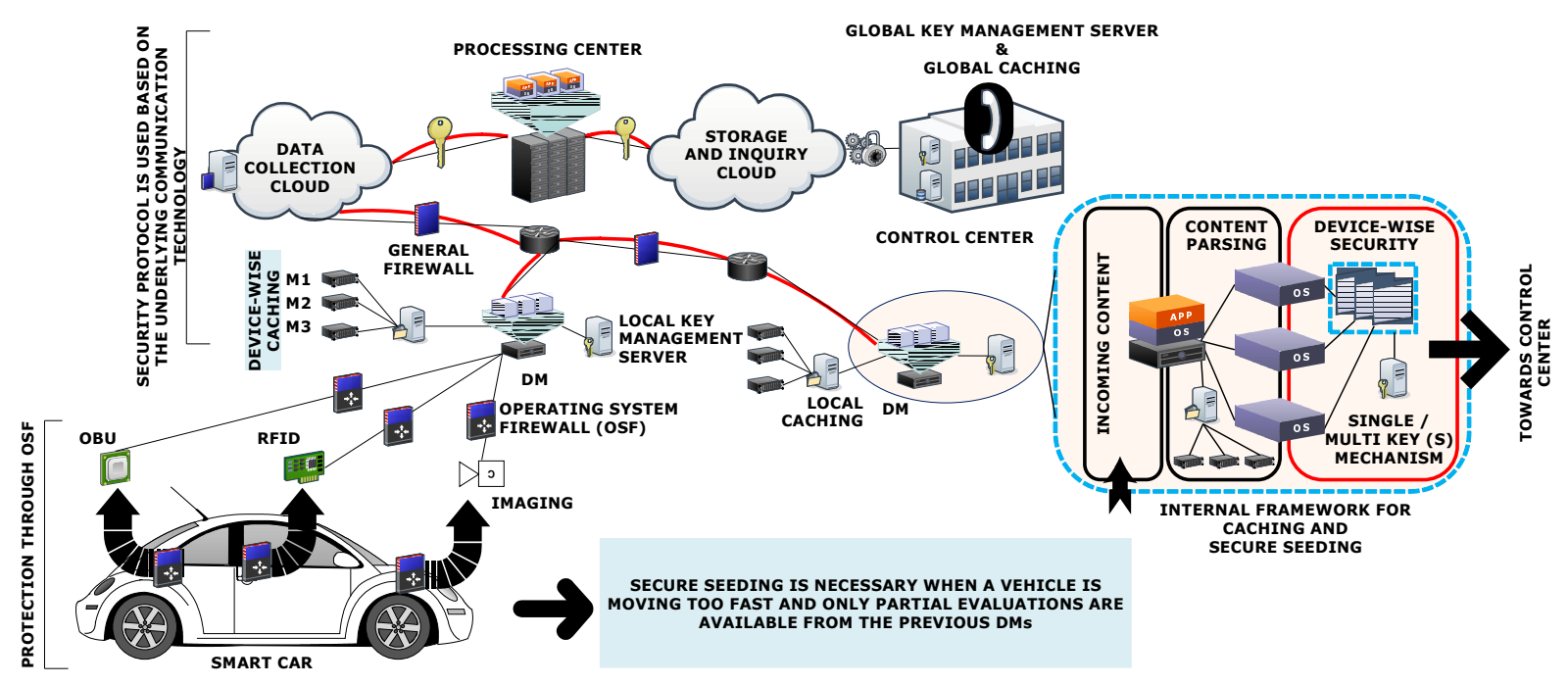

Fig. 4: An illustration of caching and secure seeding facilities for the proposed IVS.

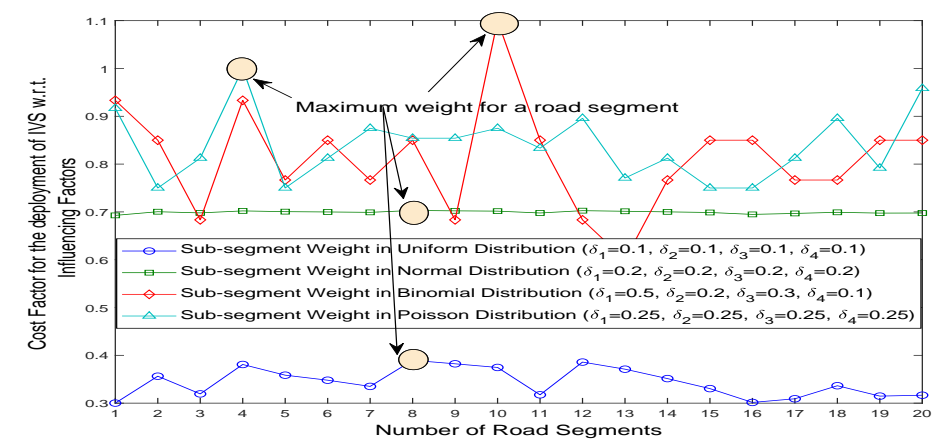

Fig. 5: Cost Factor for the deployment of the proposed system w.r.t. influencing factor. The result sets light source as a dominating factor and presents the variation of road segments according to different probability functions. $\delta_{i}$ is the corresponding priority of influencing factors.

general overview and an internal framework for caching and secure seeding in the proposed IVS are presented in Fig. 4. The secure seeding refers to securing the general transmissions as well as the caching data between the end to end connections, i.e., between the OBU and the control center. The initial key distributions for general traffic can be done through the Authentication Server placed at the control center, whereas the caching data can be secured through the derivative keys generated by the local key management servers at the respective DMs. In the hierarchy, the local key management servers act as descendent to the global key management server. The device security and exchanges between the DM and the OBU are further secured through a dedicated Operating System Firewall (OSF).

\section{RS-VH DEPLOYMENT COST FACTOR}

The efficiency of ITS is greatly influenced by the locations of RS-VH nodes in IVS [13]. Special attention must be given to the deployment of RS$\mathrm{VH}$ nodes to cover the entire city so that a maximum number of vehicles can be served without any change in the limits of approved budget $A_{B u d}$ which not only include the deployment cost but also the manufacturing cost of the RS-VH. Some of the prime factors which influence the decision of location identification are road type, segment length, light source, accident or crime frequency and an average number of vehicles.

Under the given framework of optimization [4], the Knapsack algorithm can be used to identify the potential locations for RS-VH [14]. A general evaluation of the optimization issue, in Fig. 5, is 


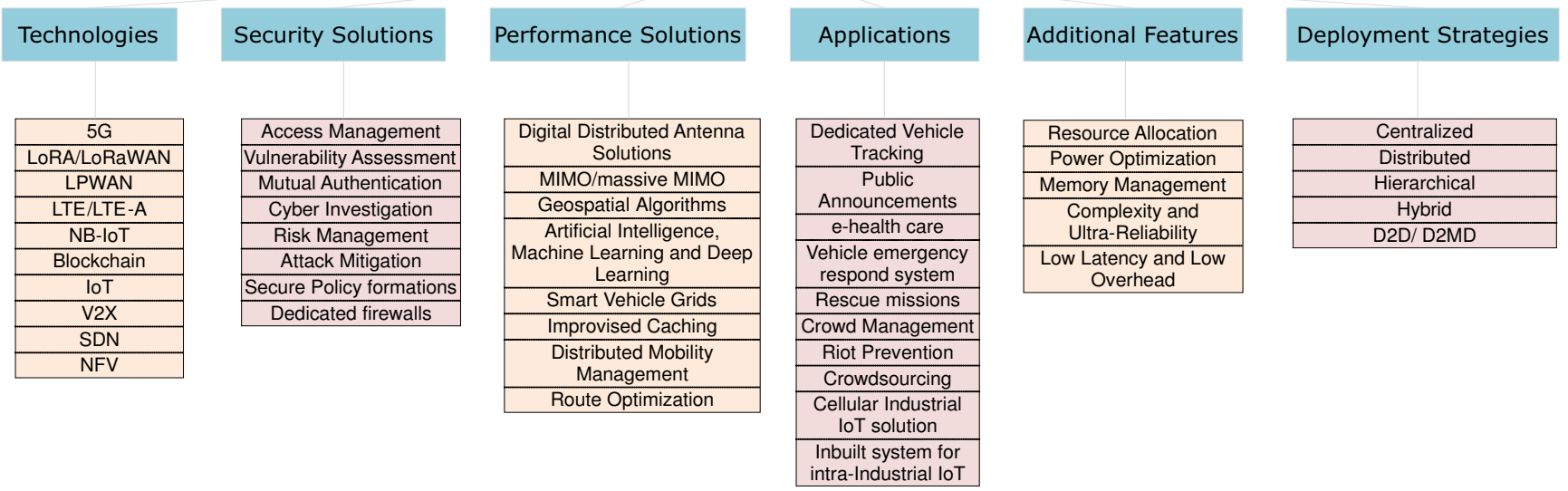

Fig. 6: An illustration representing the details of various aspects for implementing futuristic modules for the proposed IVS.

presented as a cost factor required for deploying the proposed IVS w.r.t. varying distributions of influencing factors. This factor helps to identify how much the performance will be impacted and how much will be a burden to use the proposed system with other available technologies. The cost factor can be used as a direct multiplier of any parameter to evaluate the performance of the proposed system. It further highlights that the weight and the priority associated with a particular parameter directly influence the cost of deployment.

\section{Open Research Issues And Future DIRECTIONS}

The majority of technology enablers, security aspects, performance solutions, applications, deployment strategies, and additional features that can be incorporated with the proposed IVS are presented in Fig. 6. Alongside, there are various open research challenges that need to be resolved for further enhancing the efficiency of the proposed IVS both in terms of computation time, cost, and data security. Some of which are listed below:

Privacy preservation: Although our cities are getting smarter day-by-day with technological advancement, the chances of vulnerability to private information leakage are also increasing. During capture, the transfer or processing phase, private information about user identity and locations can be accessed by an attacker. Even inside threats like an employee, agent or housekeeping staff can steal a user's data or provide entry for an outside attacker. Techniques like anonymity, encryption, and access control can be applied to save the data but these affect efficiency in terms of operational time. Therefore, in order to best manage the entire system, a balance between privacy and efficiency needs to be resolved. [15].

Parallel processing: The data generated by RS$\mathrm{VH}$ nodes will increase at an exponential rate due to a large increase in vehicular traffic. As a result, the length of the processing queue and waiting time will be increased by a large amount which is undesirable in an ITS. The bottleneck is not only at the processing center but also in the data storage cloud. Parallel processing techniques can provide a solution to the problem, but in the cloud, parallel data access remains a big challenge.

Energy management: The growing popularity of ITS applications has increased the demand for deployment of RS-VH nodes in the city which results in the consumption of more energy units. It is very difficult to fulfill the increased demand due to the limited availability of energy resources. Because of which, one of the goals of the smart city is to make the city self-reliant. Therefore, some natural energy resources like solar power must be used for the RS-VH or energy management must be done by following smart grid solutions. Identification of decision factors for efficient energy management without any decline in the performance of IVS is 
always a challenging task.

Reliability of data: Vigilare systems in smart cities may encounter a scenario involving one or more sensors/RFID Tags/ readers/cameras that may not be working at a particular time. Such issues can be encountered by switching between alternative modes of data collection if one is not available. However, identification of failing devices and additional efforts for preemptive policies to identify operational failures need to be resolved in multifaceted setups.

There is a gap to identify the correlations between the cost of deployment of efficient vigilant solutions and the economic strengths of a country. Specifically, it would be interesting to know in further reports how higher standards of living are related to the desire of building smart cities and what impact of research-investments have on deploying these technologies.

\section{COnClusion}

In this article, a solution designated multifaceted IVS is proposed to resolve the quandaries that obstruct developing countries from adopting the full-fledged utilization of ITS. Data accumulated in IVS can be utilized by the government and private agencies to make warnings and take decisions for solving a variety of ITS issues. Security of the smart city is always the highest priority issue to be tackled within ITS and has been discussed in this article, as safety is one of the main driving forces for the conception of a smart city. The deployment strategy to identify potential locations for the RS-VH and some prominent research challenges are additionally discussed.

\section{REFERENCES}

[1] I. Yaqoob, I. A. T. Hashem, Y. Mehmood, A. Gani, S. Mokhtar, and S. Guizani, "Enabling communication technologies for smart cities," IEEE Communications Magazine, vol. 55, no. 1, pp. 112-120, 2017.

[2] S. Lee, G. Tewolde, and J. Kwon, "Design and implementation of vehicle tracking system using GPS/GSM/GPRS technology and smartphone application," in Internet of Things (WF-IoT), pp. 353-358, 2014.

[3] Z. Zhou, H. Yu, C. Xu, Y. Zhang, S. Mumtaz, and J. Rodriguez, "Dependable Content Distribution in D2D-Based Cooperative Vehicular Networks: A Big Data-Integrated Coalition Game Approach," IEEE Transactions on Intelligent Transportation Systems, vol. 19, pp. 953-964, March 2018.

[4] H. Menouar, I. Guvenc, K. Akkaya, A. S. Uluagac, A. Kadri, and A. Tuncer, "UAV-enabled intelligent transportation systems for the smart city: Applications and challenges," IEEE Communications Magazine, vol. 55, no. 3, pp. 22-28, 2017.

[5] Y. Liu, X. Weng, J. Wan, X. Yue, H. Song, and A. V. Vasilakos, "Exploring data validity in transportation systems for smart cities," IEEE Communications Magazine, vol. 55, no. 5, pp. 2633, 2017.

[6] R. F. Atallah, C. M. Assi, and M. J. Khabbaz, "Scheduling the Operation of a Connected Vehicular Network Using Deep Reinforcement Learning," IEEE Transactions on Intelligent Transportation Systems, pp. 1-14, 2018.

[7] M. M. Islam, M. A. Razzaque, M. M. Hassan, W. N. Ismail, and B. Song, "Mobile cloud-based big healthcare data processing in smart cities," IEEE Access, vol. 5, pp. 11887-11899, 2017.

[8] K. Y. Chan, T. S. Dillon, and E. Chang, "An intelligent particle swarm optimization for short-term traffic flow forecasting using on-road sensor systems," IEEE Transactions on Industrial Electronics, vol. 60, no. 10, pp. 4714-4725, 2013.

[9] K. Nellore and G. P. Hancke, "A survey on urban traffic management system using wireless sensor networks," Sensors, vol. 16, no. 2, p. 157, 2016.

[10] P. Neirotti, A. De Marco, A. C. Cagliano, G. Mangano, and F. Scorrano, "Current trends in Smart City initiatives: Some stylised facts," Cities, vol. 38, pp. 25-36, 2014.

[11] A. Almagambetov, S. Velipasalar, and M. Casares, "Robust and computationally lightweight autonomous tracking of vehicle taillights and signal detection by embedded smart cameras," IEEE Transactions on Industrial Electronics, vol. 62, no. 6, pp. 3732-3741, 2015.

[12] H. Li and C. Shen, "Reading car license plates using deep convolutional neural networks and LSTMs," arXiv preprint, vol. arXiv:1601.05610, pp. 1-17, 2016.

[13] X. Fan, B. Yang, R. Yamamoto, and Y. Tanaka, "Road side unit assisted stochastic multi-hop broadcast scheme for instant emergency message propagation," in 17th International Conference on Advanced Communication Technology (ICACT), IEEE, pp. 450-457, 2015.

[14] M. B. Brahim, W. Drira, and F. Filali, "Roadside units placement within city-scaled area in vehicular ad-hoc networks," in International Conference on Connected Vehicles and Expo (ICCVE), IEEE, pp. 1010-1016, 2014.

[15] K. Zhang, J. Ni, K. Yang, X. Liang, J. Ren, and X. S. Shen, "Security and privacy in smart city applications: Challenges and solutions," IEEE Communications Magazine, vol. 55, no. 1, pp. 122-129, 2017. 